\title{
A NEW CLASS OF STRONG UNIQUENESS POLYNOMIALS SATISFYING FUJIMOTO'S CONDITION
}

\author{
Abhijit Banerjee \\ University of Kalyani, Department of Mathematics \\ West Bengal 741235, India; abanerjee_kal@yahoo.co.in \\ The author dedicates the paper to the memory of his maternal \\ grandmother Late Tripti Roy whom the author misses very much.
}

\begin{abstract}
The purpose of the paper is to introduce a new class of strong uniqueness polynomial satisfying Fujimoto's conditions. We shall also show that for a suitably prescribing value of the constant term in the class of polynomials, an SUPM (SUPE) with smallest degree ever can be obtained. We also find corresponding class of unique range sets generated from those of polynomials under weaker sharing hypothesis. Finally, rectifying the results in the application part of [2] we shall reduce the cardinalities of the range sets under some assumptions on the deficiencies of the functions. At the last section we shall place an open question for future research.
\end{abstract}

\section{Introduction, definitions and results}

In this paper we adopt the standard notations of the Nevanlinna theory of meromorphic functions as explained in [9]. Throughout the paper by a meromorphic functions we shall always mean meromorphic functions in the complex plane. It will be convenient to let $E$ denote any set of positive real numbers of finite linear measure, not necessarily the same at each occurrence. For any non-constant meromorphic function $h(z)$ we denote by $S(r, h)$ any quantity satisfying

$$
S(r, h)=o(T(r, h)) \quad(r \longrightarrow \infty, r \notin E) .
$$

For $a \in \mathbf{C} \cup\{\infty\}$, we define

$$
\Theta(a ; f)=1-\limsup _{r \rightarrow \infty} \frac{\bar{N}(r, a ; f)}{T(r, f)} .
$$

Let $f$ and $g$ be two non-constant meromorphic functions and let $a$ be a finite complex number. We say that $f$ and $g$ share the value $a \mathrm{CM}$ (counting multiplicities), provided that $f-a$ and $g-a$ have the same zeros with the same multiplicities. Similarly, we say that $f$ and $g$ share the value $a$-IM (ignoring multiplicities), provided that $f-a$ and $g-a$ have the same set of zeros, where the multiplicities are not taken into account. In addition we say that $f$ and $g$ share $\infty$ CM (IM), if $1 / f$ and $1 / g$ share $0 \mathrm{CM}(\mathrm{IM})$.

Let $S$ be a set of distinct elements of $\mathbf{C} \cup\{\infty\}$ and $E_{f}(S)=\bigcup_{a \in S}\{z: f(z)=a\}$, where each zero is counted according to its multiplicity. If multiplicities are not

doi:10.5186/aasfm.2015.4025

2010 Mathematics Subject Classification: Primary 30D35.

Key words: Meromorphic functions, uniqueness, strong uniqueness polynomial, unique range set.

The author is thankful to DST-PURSE programme for financial assistance. 
counted, then the set $\bigcup_{a \in S}\{z: f(z)=a\}$ is denoted by $\bar{E}_{f}(S)$. If $E_{f}(S)=E_{g}(S)$ we say that $f$ and $g$ share the set $S$ CM. On the other hand, if $\bar{E}_{f}(S)=\bar{E}_{g}(S)$, we say that $f$ and $g$ share the set $S$ IM. Evidently, if $S$ contains only one element, then it coincides with the usual definition of CM (respectively, IM) sharing of values.

Let $l$ be a positive integer or infinity. We denote by $E_{l)}(a, f)$ the set of $a$-points of $f$ whose multiplicities are not greater than $l$ and each $a$-point is counted according to its multiplicity. For $S \subset \mathbf{C} \cup\{\infty\}$ we put $E_{l)}(S, f)=\bigcup_{a \in S} E_{l)}(a ; f)$.

In 1926, R. Nevanlinna discovered his famous five value uniqueness theorem which says that if two non-constant meromorphic functions $f$ and $g$ on the complex plane C share five distinct values IM then $f \equiv g$. A few years later, he showed that when multiplicities are considered, 4 points are sufficient to determine the functions and in this case either the functions coincide or one is the bilinear transformation of the other. These two results can be considered as the backbone of the present uniqueness theory which has been reached at the pinnacle of prosperity specially during the last two decades.

In [7] Gross extended the study of uniqueness by determining an entire function with the help of single pre-image of a finite set $S$ counting multiplicities. In 1982 Gross and Yang [8] proved the following theorem:

Theorem A. [8] Let $S=\left\{z \in \mathbf{C}: e^{z}+z=0\right\}$. If two entire functions $f, g$ satisfy $E_{f}(S)=E_{g}(S)$, then $f \equiv g$.

In [8], the definition of unique range set for entire function was first introduced which could easily be re-iterated for meromorphic functions as well. Below we are recalling the same.

Let a set $S \subset \mathbf{C}$ and $f$ and $g$ be two non-constant meromorphic (entire) functions. If $E_{f}(S)=E_{g}(S)$ implies $f \equiv g$ then $S$ is called a unique range set for meromorphic (entire) functions or in brief URSM (URSE).

In 1997, Yi [15] introduced the analogous definition for reduced unique range sets. We will call any set $S \subset \mathbf{C}$ a unique range set for meromorphic (entire) functions ignoring multiplicity (URSM-IM) (URSE-IM) or a reduced unique range set for meromorphic (entire) functions (RURSM) (RURSE) if $\bar{E}_{f}(S)=\bar{E}_{g}(S)$ implies $f \equiv g$ for any pair of non-constant meromorphic (entire) functions.

It is to be observed that since the range set $S$ given in Theorem A is an infinite set, Theorem A cannot be considered as an exact solution to the problem of Gross.

After the introduction of the novel idea of unique range sets researchers were getting more involved to find new unique range sets with cardinalities as small as possible. In 1994, Yi [13] exhibited a URSE with 15 elements and in $1995 \mathrm{Li}$ and Yang [10] exhibited a URSM with 15 elements and a URSE with 7 elements. Till date the URSM with 11 elements are the smallest available URSM obtained by Frank and Reinders [4]. The URSM discovered by Frank and Reinders is highlighted by a number of researchers.

$\mathrm{Li}$ and Yang [10] first pointed out the fact that the finite URSM's are the set of distinct zeros of some suitable polynomials and so one cannot deny the importance of the underlying polynomial.

According to Li and Yang [10], a polynomial $P$ in $\mathbf{C}$, is called a uniqueness polynomial for meromorphic (entire) functions, if for any two non-constant meromorphic 
(entire) functions $f$ and $g, P(f) \equiv P(g)$ implies $f \equiv g$. We say $P$ is a UPM (UPE) in brief.

On the other hand, An, Wang and Wong [1] called a polynomial $P$ in $\mathbf{C}$ as a strong uniqueness polynomial for meromorphic (entire) functions if for any nonconstant meromorphic (entire) functions $f$ and $g, P(f) \equiv c P(g)$ implies $f \equiv g$, where $c$ is a suitable nonzero constant. In this case we say $P$ is a SUPM (SUPE) in brief.

Following two theorems may be considered as the initial characterisations of a uniqueness polynomial.

Theorem B. [10] Any polynomial of degree 2 or 3 is not a UPE.

Theorem C. [10] Let $P(z)=z^{4}+a_{3} z^{3}+a_{2} z^{2}+a_{1} z+a_{0}$. Then $P$ is not a UPM. Also $P$ is a UPE if and only if $\left(\frac{a_{3}}{2}\right)^{3}-\frac{a_{2} a_{3}}{2}+a_{1} \neq 0$.

The polynomials of higher degree require further considerations, which first studied by Yang and Hua [12].

However in [5], to find a necessary and sufficient condition for a monic polynomial without multiple zero to be a UPM, Fujimoto manipulated the polynomial in a different way. Let $P(z)$ be a monic polynomial without multiple zero whose derivatives has mutually distinct $k$ zeros given by $d_{1}, d_{2}, \ldots, d_{k}$ with multiplicities $q_{1}, q_{2}, \ldots, q_{k}$ respectively. The following theorem of Fujimoto helps us to find many uniqueness polynomials in which the term "critically injective" is used which has been explained after Example 1.2.

Theorem D. [6] Suppose that $P(z)$ is critically injective. Then $P(z)$ will be a uniqueness polynomial if and only if

$$
\sum_{1 \leq l<m \leq k} q_{l} q_{m}>\sum_{l=1}^{k} q_{l} .
$$

In particular the above inequality is always satisfied whenever $k \geq 4$. When $k=3$ and $\max \left\{q_{1}, q_{2}, q_{3}\right\} \geq 2$ or when $k=2, \min \left\{q_{1}, q_{2}\right\} \geq 2$ and $q_{1}+q_{2} \geq 5$, then also the above inequality holds.

For $k=1$, taking $P(z)=(z-a)^{q}-b$ for some constants $a$ and $b$ with $b \neq 0$ and an integer $q \geq 2$, it is easy to verify that for an arbitrary non-constant meromorphic function $g$ and a constant $c(\neq 1)$ with $c^{q}=1$, the function $g:=c f+(1-c) a(\neq f)$ satisfies the condition $P(f)=P(g)$.

Let $P$ be a polynomial of degree $n$ in $\mathbf{C}$ having only simple zeros and $S$ be the set of all zeros of $P$. If $S$ is a URSM (URSE), then from the definition it follows that $P$ is a UPM (UPE). However from the following examples given in [3] and [12], it is evident that the converse is not true.

Example 1.1. [3] Let $P(z)=a z+b(a \neq 0)$. Clearly $P(z)$ is a UPM but for $f=-\frac{b}{a} e^{z}$ and $g=-\frac{b}{a} e^{-z}$ we see that $E_{f}(S)=E_{g}(S)$, where $S=\left\{-\frac{b}{a}\right\}$ is the set of zeros of $P(z)=a z+b$.

Example 1.2. [12] Let $P(z)=z^{4}+2 z^{3}-9 z^{2}-2 z+8=(z-1)(z+1)(z-2)(z+4)$. By Theorem C, $P(z)$ is a UPE. Here $S=\{1,-1,2,4\}$. However, for two different entire functions $f(z)=\frac{3}{2} \sqrt{5} e^{z}+\frac{7}{2}$ and $g(z)=\frac{3}{2} \sqrt{5} e^{-z}+\frac{7}{2}$, one can easily check $E_{f}(S)=E_{g}(S)$. 
Therefore it would be really interesting to investigate under which additional assumption the converse situation will hold good.

In this connection, Fujimoto [5] first discovered a special property of a polynomial, reasonably called as critical injection property in [3] though initially Fujimoto called it as property $(\mathrm{H})$. Critical injection property of a polynomial may be stated as follows: A polynomial $P$ is said to satisfy critical injection property if $P(\alpha) \neq P(\beta)$ for any two distinct zeros $\alpha, \beta$ of the derivative $P^{\prime}$.

Clearly the meaning of critical injection property is that the polynomial $P$ is injective on the set of distinct zeros of $P^{\prime}$, which are known as critical points of $P$. Naturally a polynomial with this property may be called a critically injective polynomial. Fujimoto showed that the critical injection property of polynomial helps one to find a sufficient condition for a set of zeros $S$ of even a SUPM (SUPE) $P$ to be a URSM (URSE).

Theorem E. [5] Let $P(z)=\left(z-\alpha_{1}\right)\left(z-\alpha_{2}\right) \ldots\left(z-\alpha_{n}\right)$ be a critically injective polynomial of degree $n$ in $\mathbf{C}$ having only simple zeros. Let $P^{\prime}$ have $k$ distinct zeros and either $k \geq 3$ or $k=2$ and $P^{\prime}$ have no simple zero. Further suppose that $P$ is a SUPM (SUPE). If $S$ is the set of zeros of $P$, then $S$ is a URSM (URSE) whenever $n>2 k+6(n>2 k+2)$ while a URSM-IM (URSE-IM) whenever $n>2 k+12$ $(n>2 k+5)$.

Recently the definition of unique range sets have been generalized in [3] as follows:

Definition 1.1. [3] A set $S$ is called a $\mathrm{URSM}_{l)}\left(\mathrm{URSE}_{l}\right)$ ) if for any two nonconstant meromorphic (entire) functions $f, g, E_{l)}(S, f)=E_{l)}(S, g)$ implies $f \equiv g$.

In 2009 Bai, Han and Chen [2] proved the following truncated sharing version of Theorem E.

Theorem F. [2] In addition to the hypothesis of Theorem $E$ we suppose that $l$ is a positive integer or $\infty$. Let $S$ be the set of zeros of $P$. If

(i) $l \geq 3$ or $\infty$ and $n>2 k+6(2 k+2)$,

(ii) $l=2$ and $n>2 k+7(2 k+2)$,

(iii) $l=1$ and $n>2 k+10(2 k+4)$,

then $S$ is a $\mathrm{URSM}_{l)}\left(\mathrm{URSE}_{l)}\right)$.

We recall the URSM introduced by Frank and Reinders [4] which is the zero set of

$$
P_{F R}(z)=\frac{(n-1)(n-2)}{2} z^{n}-n(n-2) z^{n-1}+\frac{n(n-1)}{2} z^{n-2}-c \quad(c \neq 0,1) .
$$

Clearly $P_{F R}^{\prime}(z)$ has two distinct zeros that is here $k=2$. From [4], we know $P_{F R}$ is a UPM if $n \geq 6$. Also from [4, p. 191, Case 2] it is clear that whenever $n \geq 8$, $P_{F R}(f) \equiv c P_{F R}(g)$ implies $P_{F R}(f) \equiv P_{F R}(g)$ that is $P_{F R}$ is a SUPM satisfying critical injection property when $n \geq 8$. Hence if we denote the zero set of $P_{F R}(z)$ as $S_{F R}$ then $S_{F R}$ becomes a $\mathrm{URSM}_{l}\left(\mathrm{URSE}_{l)}\right)$ for the cases $l \geq 3$ or $\infty, l=2$ and $l=1$ when it contains 11, 12, 15 elements (respectively 7, 7, 9 elements).

Second type of URSM is demonstrated by Yi in [14] which is the zero set of

$$
P_{Y}(z)=z^{n}+a z^{n-r}+b,
$$

where $n, r$ are two positive integers having no common factors, $r \geq 2$ and $a$ and $b$ are so chosen so that $P$ has $n$ distinct zeros. Here $k=r+1$ and $P_{Y}$ is a UPM if 
$n \geq 7$, see [14, p. 79, Case 3, last part]. Also from [14, p. 79, Case 3, first part] it is clear that whenever $n \geq 2 r+5, P_{Y}(f) \equiv c P_{Y}(g)$ implies $P_{Y}(f) \equiv P_{Y}(g)$ and hence it is a critically injective SUPM. Hence if we denote the zero set of $P_{Y}(z)$ as $S_{Y}$, then $S_{Y}$ becomes a $\mathrm{URSM}_{l)}\left(\mathrm{URSE}_{l)}\right)$ for the cases $l \geq 3$ or $\infty, l=2$ and $l=1$ when it contains $2 r+9,2 r+10,2 r+13$ elements (respectively $2 r+5,2 r+5,2 r+7$ elements).

So we observe that the smallest degree of critically injective SUPM discovered ever is $P_{F R}(z)$ when $n \geq 8$. To reduce the cardinalities of the range sets further in the application part of [2] the following theorem was proved.

Theorem G. [2] In addition to the hypothesis of Theorem $F$ we suppose that $l$ is a positive integer or $\infty$. Let $S$ be the set of zeros of $P$. If

(i) $l \geq 3$ or $\infty$ and $\Theta(\infty ; f)+\Theta(\infty ; g)>3+k-\frac{n}{2}$,

(ii) $l=2$ and $\Theta(\infty ; f)+\Theta(\infty ; g)>\frac{28+8 k-4 n}{9}$,

(iii) $l=1$ and $\Theta(\infty ; f)+\Theta(\infty ; g)>\frac{20+4 k-2 n}{6}$, then $S$ is a $\mathrm{URSM}_{l)}$.

The proof of the above theorem is not flawless. For example if we carefully observe third line after (6.1) of p. 641, in the proof of [2, Theorem 6.1] we see that the equation have been calculated on the basis of the assumption that

$$
T(r) \leq 2 T_{0}(r) \Rightarrow(2 k-n+2) T(r)(=T(r, f)+T(r, g)) \leq 2(2 k-n+2) T_{0}(r)
$$

which is only true when $2 k+2 \geq n$. But then [2, Theorem 6.1] is not correct since for all the cases with $l \geq 3$ or $\infty, l=2$ and $l=1$, we have from Theorem G, $\Theta(\infty ; f)+\Theta(\infty ; g)>2$, which is impossible.

With the help of Theorem $G$ in [2], investigations on further reduction of the cardinalities of $S_{F R}$ were carried out. As we have already shown that Theorem G is not correct, it would be better to find first its rectified form than to ponder over its applications.

In the paper we introduce a new class of SUPM which is also critically injective but different from $P_{F R}$. We shall also show that for a suitable choice of the constant term in our polynomial it will be a SUPM (SUPE) with smaller degree than that of Frank and Reinders. Finally, we will rectify Theorem G and investigate its implications on the SUPM $P_{F R}$ and the same obtained by us. Henceforth for two positive integers $n, m$ we shall denote by $P(z)$ the following polynomial

$$
P(z)=\sum_{i=0}^{m}\left(\begin{array}{c}
m \\
i
\end{array}\right) \frac{(-1)^{i}}{n+m+1-i} z^{n+m+1-i}+c=Q(z)+c,
$$

where

$$
Q(z)=\sum_{i=0}^{m}\left(\begin{array}{c}
m \\
i
\end{array}\right) \frac{(-1)^{i}}{n+m+1-i} z^{n+m+1-i}, \quad c \neq 0,
$$

is a constant. Clearly $P^{\prime}(z)=z^{n}(z-1)^{m}$. So $P(0)=c$ and $P(1)=Q(1)+c$.

Following theorem is the main result of the paper.

Theorem 1.1. Let $n, m \geq 3(m \geq 2)$ be two positive integers. Now

(I) when $c \neq 1, n \geq m+3(n \geq m+2)$, or

(II) when $c=1, n \geq 3(n \geq 2)$, then $P$ given by (1.1), is a SUPM (SUPE).

Remark 1.1. From Theorem 1.1, it is clear that when $c \neq 1$ and $n+m \geq 9$ $(n+m \geq 6)$, i.e., when degree of the polynomial is at-least 10 (7) or when $c=1$ and 
$n+m \geq 6(\min \{m, n\} \geq 2$, with $m+n \geq 5)$, i.e., when degree of the polynomial is at least 7 (6), then $P$ given by (1.1) is a SUPM (SUPE).

Remark 1.2. When $c=1,(1.1)$ produce a new kind of critically injective SUPM (SUPE) with degree 7 (6). Till date this is the smallest degree critically injective SUPM (SUPE) ever introduced.

Theorem 1.2. Let $n, m(\geq 3)$ be two positive integers. We suppose that $S=$ $\{z: P(z)=0\}$. Now

(I) when $c \neq 1, n \geq m+3$, or

(II) when $c=1, n \geq 3$ and one of the following conditions holds:

(a) $l \geq 3$ or $\infty$ and $n+m \geq 10$ (6),

(b) $l=2$ and $n+m \geq 11(6)$,

(c) $l=1$ and $n+m \geq 15(9)$,

then $S$ is a $\mathrm{URSM}_{l)}\left(\mathrm{URSE}_{l)}\right)$.

\section{Lemma}

The following lemma is needed to proceed further.

Lemma 2.1. [11] Let $f$ be a non-constant meromorphic function and let

$$
R(f)=\frac{\sum_{k=0}^{n} a_{k} f^{k}}{\sum_{j=0}^{m} b_{j} f^{j}}
$$

be an irreducible rational function in $f$ with constant coefficients $\left\{a_{k}\right\}$ and $\left\{b_{j}\right\}$ where $a_{n} \neq 0$ and $b_{m} \neq 0$. Then

$$
T(r, R(f))=d T(r, f)+S(r, f),
$$

where $d=\max \{n, m\}$.

Lemma 2.2. $Q(1)$ is not an integer. In particular, when $c=1, P(1) \neq-1$, where $n \geq 3, m \geq 3$ are integers.

Proof. We claim that

$$
\begin{aligned}
S_{n}(m) & =\sum_{i=0}^{m}\left(\begin{array}{c}
m \\
i
\end{array}\right) \frac{(-1)^{i}}{n+m+1-i} \\
& =\frac{\left(\begin{array}{c}
m \\
0
\end{array}\right)}{n+m+1}-\frac{\left(\begin{array}{c}
m \\
1
\end{array}\right)}{n+m+1-1}+\ldots+(-1)^{m} \frac{\left(\begin{array}{c}
m \\
m
\end{array}\right)}{n+1} \\
& =\frac{(-1)^{m} m !}{(n+m+1)(n+m) \ldots(n+1)} .
\end{aligned}
$$

We prove the claim by method of induction on $m$.

At first for $m=3$ we get

$$
S_{n}(3)=\frac{1}{n+4}-\frac{3}{n+3}+\frac{3}{n+2}-\frac{1}{n+1}=\frac{(-1)^{3} \cdot 3 !}{(n+4)(n+3)(n+2)(n+1)} .
$$

So, $S_{n}(m)$ is true for $m=3$. Now we assume that $S_{n}(m)$ is true for $m=k$, where $k$ is any given positive integer such that $k \geq 3$. We will show that $S_{n}(m)$ is true for 
$m=k+1$, i.e.,

$$
\frac{\left(\begin{array}{c}
k+1 \\
0
\end{array}\right)}{n+k+2}-\frac{\left(\begin{array}{c}
k+1 \\
1
\end{array}\right)}{n+k+1}+\ldots+(-1)^{k+1} \frac{\left(\begin{array}{c}
k+1 \\
k+1
\end{array}\right)}{n+1}=\frac{(-1)^{(k+1)}(k+1) !}{(n+k+2)(n+k+1) \ldots(n+1)} .
$$

Using induction hypothesis and noting that $\left(\begin{array}{c}k+1 \\ i\end{array}\right)=\left(\begin{array}{c}k \\ i\end{array}\right)+\left(\begin{array}{c}k \\ i-1\end{array}\right)$ for $i \geq 1$, we have

$$
\begin{aligned}
S_{n}(k+1) & =\frac{\left(\begin{array}{l}
k \\
0
\end{array}\right)}{n+k+2}-\frac{\left(\begin{array}{l}
k \\
0
\end{array}\right)+\left(\begin{array}{l}
k \\
1
\end{array}\right)}{n+k+1}+\frac{\left(\begin{array}{l}
k \\
1
\end{array}\right)+\left(\begin{array}{l}
k \\
2
\end{array}\right)}{n+k}-\ldots+(-1)^{k} \frac{\left(\begin{array}{c}
k \\
k-1
\end{array}\right)+\left(\begin{array}{l}
k \\
k
\end{array}\right)}{n+2}+(-1)^{k+1} \frac{\left(\begin{array}{l}
k \\
k
\end{array}\right)}{n+1} \\
= & {\left[\frac{\left(\begin{array}{l}
k \\
0
\end{array}\right)}{(n+1)+k+1}-\frac{\left(\begin{array}{l}
k \\
1
\end{array}\right)}{(n+1)+k}+\frac{\left(\begin{array}{l}
k \\
2
\end{array}\right)}{(n+1)+k-1}-\ldots+(-1)^{k} \frac{\left(\begin{array}{l}
k \\
k
\end{array}\right)}{(n+1)+1}\right] } \\
& -\left[\frac{\left(\begin{array}{l}
k \\
0
\end{array}\right)}{n+k+1}-\frac{\left(\begin{array}{l}
k \\
1
\end{array}\right)}{n+k}+\frac{\left(\begin{array}{l}
k \\
2
\end{array}\right)}{n+k-1}-\ldots+(-1)^{k-1} \frac{\left(\begin{array}{c}
k \\
k-1
\end{array}\right)}{n+2}+(-1)^{k} \frac{\left(\begin{array}{l}
k \\
k
\end{array}\right)}{n+1}\right] \\
= & \frac{S_{n+1}(k)-S_{n}(k)}{(n+k+2)(n+k+1) \ldots(n+2)}-\frac{(-1)^{k} k !}{(n+k+1)(n+k) \ldots(n+1)} \\
= & \frac{(-1)^{(k+1)}(k+1) !}{(n+k+2)(n+k+1) \ldots(n+1)} .
\end{aligned}
$$

So our claim has been established. We note that $S_{n}(m)=(-1)^{m} \prod_{i=1}^{m} \frac{i}{(n+i)} \frac{1}{(n+m+1)}$ and hence it can not be an integer. In particular we have proved that $Q(1) \neq-2$, i.e., when $c=1, P(1) \neq-1$.

\section{Proofs of the theorems}

Proof of Theorem 1.1. Let $F$ and $G$ be two non-constant meromorphic functions given by $F=P(f)$ and $G=P(g)$. First we observe that by Lemma $2.2, Q(1) \neq 0$. Since $P(0)=c \neq P(1)=Q(1)+c, P(z)$ is critically injective polynomial. Also $P(z)-c$ and $P(z)-P(1)$ have a zero of multiplicity $n+1$ and $m+1$ respectively at 0 and 1 , it follows that $P(z)$ has simple zeros. Let the zeros be given by $\alpha_{j}$, $j=1,2, \ldots, n+m+1$. Suppose

$$
P(f) \equiv A P(g)
$$

$$
F \equiv A G
$$

From (3.1) using Lemma 2.1 we have

$$
T(r, f) \equiv T(r, g)+S(r, g) .
$$

Case 1. Suppose $A \neq 1$.

Subcase 1.1. Assume $A=P(1)$, then from (3.2) we have

$$
F-P(1) \equiv P(1)(G-1) \text {. }
$$

Suppose $c \neq 1$, it follows that all the zeros of $P(z)-1$ are simple and let them be $\beta_{i}, i=1,2, \ldots, n+m+1$. Clearly $P(z)-P(1)$ has a zero at 1 of order $m+1$. Let 
the remaining distinct zeros of $P(z)-P(1)$ be $\gamma_{j}, j=1,2, \ldots, n$. Then

$$
\bar{N}(r, 1 ; f)+\sum_{j=1}^{n} \bar{N}\left(r, \gamma_{j} ; f\right)=\sum_{i=1}^{n+m+1} \bar{N}\left(r, \beta_{i} ; g\right) .
$$

So from the second fundamental theorem and (3.3) we get

$$
\begin{aligned}
(n+m-1) T(r, g) & \leq \sum_{i=1}^{n+m+1} \bar{N}\left(r, \beta_{i} ; g\right)+S(r, g) \\
& \leq(n+1) T(r, f)+S(r, f) \leq(n+1) T(r, g)+S(r, g),
\end{aligned}
$$

a contradiction since $m \geq 3$.

Next suppose $c=1$. Then from (3.2) we have

$$
F-1 \equiv P(1)\left(G-\frac{1}{P(1)}\right)
$$

Since $P(1) \neq 1$ and Lemma 2.2 implies $P(1) \neq-1$ we have $\frac{1}{P(1)} \neq P(1)$, it follows that $P(z)-\frac{1}{P(1)}$ has simple zeros. Let they be given by $\beta_{i}^{\prime}, i=1,2, \ldots, n+m+1$. We also let $\delta_{j}, j=1,2, \ldots, m$ be the distinct simple zeros of $P(z)-c$. So from the second fundamental theorem and (3.3) we get

$$
\begin{aligned}
(n+m-1) T(r, g) & \leq \sum_{i=1}^{n+m+1} \bar{N}\left(r, \beta_{i}^{\prime} ; g\right)+S(r, g) \\
& \leq \bar{N}(r, 0 ; f)+\sum_{j=1}^{m} \bar{N}\left(r, \delta_{j} ; f\right) \leq(m+1) T(r, g)+S(r, g),
\end{aligned}
$$

a contradiction since $n \geq 3$.

Subcase 1.2. Let $A \neq P(1)$. If $c \neq 1$, then we have from (3.2)

$$
F-A c \equiv A(G-c) .
$$

Clearly $A c \neq c$. Suppose $A c \neq P(1)$. Let the distinct zeros of $P(z)-A c$ be given by $\gamma_{i}^{\prime}, i=1,2, \ldots, n+m+1$. So from the second fundamental theorem and (3.3) we get

$$
\begin{aligned}
(n+m-1) T(r, f) & \leq \sum_{i=1}^{n+m+1} \bar{N}\left(r, \gamma_{i}^{\prime} ; f\right)+S(r, f) \\
& =\sum_{j=1}^{m} \bar{N}\left(r, \delta_{j} ; g\right)+\bar{N}(r, 0 ; g)+S(r, g) \\
& \leq(m+1) T(r, f)+S(r, f),
\end{aligned}
$$

a contradiction since $n \geq 3$. 
Next suppose $A c=P(1)$. Then again by the same way as above we get

$$
\begin{aligned}
(n-1) T(r, f) & \leq \sum_{i=1}^{n} \bar{N}\left(r, \gamma_{i} ; f\right)+\bar{N}(r, 1 ; f)+S(r, f) \\
& =\sum_{j=1}^{m} \bar{N}\left(r, \beta_{j} ; g\right)+\bar{N}(r, 0 ; g)+S(r, g) \\
& \leq(m+1) T(r, f)+S(r, f),
\end{aligned}
$$

a contradiction since $n \geq m+3$.

If $c=1$, then we have

$$
F-A \equiv A(G-1)
$$

In this case since $n \geq 3$, proceeding as above when $A c=A \neq P(1)$, we can get a contradiction. So we omit the detail.

Case 2. Suppose $A=1$. Then from (3.2) we have $F \equiv G$, i.e., $P(f) \equiv P(g)$. Here $k=2, d_{1}=0, d_{2}=1, q_{1}=n, q_{2}=m$. Also we see that $n m>n+m$, when $n \geq 3$ and $m \geq 3$. So from Theorem $\mathrm{D}$ we conclude that $P(z)$ is a uniqueness polynomial. Therefore $f \equiv g$. Hence for the case $c=1, P(z)$ is a SUPM (SUPE), when $n \geq 3, m \geq 3(\min \{m, n\} \geq 2$, with $m+n \geq 5)$ and for the case $c \neq 1, P(z)$ is a SUPM (SUPE) when $n \geq m+3, m \geq 3(n \geq m+2, m \geq 2)$. That is when $c=1$ and $P(z)$ contains at least $7(6)$ zeros or $c \neq 1$ and $P(z)$ contains at least 10 (7) zeros, it is a SUPM (SUPE). This proves the theorem.

Proof of Theorem 1.2. Since from Theorem 1.1 we know when $n+m \geq 10$ (6), $P(z)$ is an SUPM (SUPE), the theorem follows from Theorem F.

\section{Applications}

One can easily prove that the actual statement of Theorem G should be

Theorem 4.1. In addition to the hypothesis of Theorem $F$ we suppose that $l$ is a positive integer or $\infty$. Let $S$ be the set of zeros of $P$. If

(i) $l \geq 3$ or $\infty$ and $\min \{\Theta(\infty ; f), \Theta(\infty ; g)\}>\frac{6+2 k-n}{4}$,

(ii) $l=2$ and $\min \{\Theta(\infty ; f), \Theta(\infty ; g)\}>\frac{14+4 k-2 n}{9}$,

(iiii) $l=1$ and $\min \{\Theta(\infty ; f), \Theta(\infty ; g)\}>\frac{10+2 k-n}{6}$, then $S$ is a $\mathrm{URSM}_{l)}$.

From Theorem 4.1 it is clear that there exists a $\left.\mathrm{URSM}_{3}\right)\left(\mathrm{URSM}_{2)}\right)$ say $S_{F R}$ or $S$ consisting of 7 elements with the assumption $\min \{\Theta(\infty ; f), \Theta(\infty ; g)\}>\frac{3}{4}$ $\left(\min \{\Theta(\infty ; f), \Theta(\infty ; g)\}>\frac{8}{9}\right)$; while there exists an $\mathrm{URSM}_{1)}$ say $S_{F R}$ or $S$ consisting of 9 elements with the assumption $\min \{\Theta(\infty ; f), \Theta(\infty ; g)\}>\frac{5}{6}$. These are the best known lower bounds of any $\mathrm{URSM}_{l)}$.

\section{Concluding remark and an open question}

From [10] it is clear that any polynomial with degree $n, 2 \leq n \leq 5$, cannot be a UPM. So from Remark 1.2 the following question is inevitable.

Does there exist any critically injective SUPM with degree less than 7 ? 


\section{References}

[1] An, T. T. H., J. T. WAng, and P. Wong: Strong uniqueness polynomials: The complex case. - Complex Var. Theory Appl. 49:1, 2004, 25-54.

[2] Bai, X., Q. Han, and A. Chen: On a result of H. Fujimoto. - J. Math. Kyoto Univ. 49:3, 2009, 631-643.

[3] BanerJee, A., and I. LAhIRI: A uniqueness polynomial generating a unique range set and vise versa. - Comput. Methods Funct. Theory 12:2, 2012, 527-539.

[4] Frank, G., and M. Reinders: A unique range set for meromorphic functions with 11 elements. - Complex Var. Theory Appl. 37:1, 1998, 185-193.

[5] Fujimoto, H.: On uniqueness of meromorphic functions sharing finite sets. - Amer. J. Math. $122,2000,1175-1203$.

[6] Fujimoto, H.: On uniqueness polynomials for meromorphic functions. - Nagoya Math. J. 170, 2003, 33-46.

[7] Gross, F.: Factorization of meromorphic functions and some open problems. - In: Proc. Conf. Univ. Kentucky (Lexington, Kentucky, 1976), Lecture Notes in Math. 599, 1977, 51-69.

[8] Gross, F., and C. C. YAng: On preimage and range sets of meromorphic functions. - Proc. Japan Acad. 58, 1982, 17-20.

[9] Hayman, W. K.: Meromorphic functions. - The Clarendon Press, Oxford, 1964.

[10] LI, P., and C. C. YANG: Some further results on the unique range sets of meromorphic functions. - Kodai Math. J. 18, 1995, 437-450.

[11] Monon'ko, A.Z.: On the Nevanlinna characteristics of some meromorphic functions. - Teor. Funkciŭ Funkcional. Anal. i Priložen. 14, 1971, 83-87 (in Russian).

[12] YAnG, C.C., and X.H. HuA: Unique polynomials of entire and meromorphic functions. Mat. Fiz. Anal. Geom. 4:3, 1997, 391-398.

[13] YI, H. X.: On a problem of Gross. - Sci. China Ser. A 24, 1994, 1137-1144.

[14] YI, H. X.: Unicity theorems for meromorphic or entire functions III. - Bull. Austral. Math. Soc. 53, 1996, 71-82.

[15] YI, H. X.: The reduced unique range sets for entire or meromorphic functions. - Complex Var. Theory Appl. 32, 1997, 191-198. 